Research, part of a Special Feature on New Methods for Adaptive Water Management

\title{
A Framing Approach to Cross-disciplinary Research Collaboration: Experiences from a Large-scale Research Project on Adaptive Water Management
}

\author{
$\underline{\text { Art Dewulf }}^{1}, \underline{\text { Greet François }}^{1}, \underline{\text { Claudia Pahl-Wostl }}^{2}$, and $^{\text {Tharsi Taillieu }}{ }^{1}$
}

\begin{abstract}
Although cross-disciplinary research collaboration is necessary to achieve a better understanding of how human and natural systems are dynamically linked, it often turns out to be very difficult in practice. We outline a framing approach to cross-disciplinary research that focuses on the different perspectives that researchers from different backgrounds use to make sense of the issues they want to research jointly. Based on interviews, participants' evaluations, and our own observations during meetings, we analyze three aspects of frame diversity in a large-scale research project. First, we identify dimensions of difference in the way project members frame the central concept of adaptive water management. Second, we analyze the challenges provoked by the multiple framings of concepts. Third, we analyze how a number of interventions (interactive workshops, facilitation, group model building, and concrete case contexts) contribute to the connection and integration of different frames through a process of joint learning and knowledge construction.
\end{abstract}

Key Words: adaptive management; cross-disciplinary research; framing

\section{INTRODUCTION}

When researchers from different institutes and disciplines join up to study a common issue, they face a number of challenges. Communication and coordination problems, misunderstandings, and mismatched expectations easily arise. We contend that an important challenge in these crossdisciplinary endeavors is dealing with the diversity of frames or perspectives that people use to make sense of the issues of importance in a specific research context. In this paper, we outline a framing approach to cross-disciplinary research and use it to study a large-scale cross-disciplinary research project on adaptive water management.

The large-scale research project we studied is an EU-funded project, which brings together people from various nationalities, cultures, institutes, and scientific disciplines. The aim is to develop the scientific base and practical methods to implement an adaptive approach to water management, drawing on a wide range of fields such as hydrology, management, ecology, geography, systems sciences, economics, psychology, and political science. People from these different backgrounds are interdependent in performing their tasks and achieving the goal of developing a workable approach to adaptive water management. At the same time, they pursue their individual interests and want to achieve insights related to their own fields of specialization. This turns the project effectively into a multi-actor collaborative effort (Gray 1989).

Although much has been written on the necessity and benefits of cross-disciplinary research, relatively little is known about how it actually works. In this sense, cross-disciplinary research itself becomes a crucial research topic if we want to achieve a better understanding of how human and natural systems are dynamically linked. Therefore, much effort is devoted within this project to investigating and monitoring experiences within a cross-disciplinary and integrated research approach. The current paper analyzes this process over the first 18 months of a 4-year project. 
In developing a framing approach to crossdisciplinary collaboration, this paper focuses on three related issues or research questions, which each have a theoretical and an empirical aspect.

1. How can one understand frame differences theoretically in the context of crossdisciplinary collaboration, and how do frame differences manifest themselves in the studied project?

2. What challenges do these frame differences pose for research collaboration and how do they manifest themselves in the studied project?

3. How can these frame differences be dealt with constructively and how do the interventions undertaken in the studied project contribute to this?

We start with a theoretical discussion of framing and cross-disciplinary research collaboration, then outline the methods used, and finally report and discuss the results.

\section{A FRAMING VIEW ON CROSS- DISCIPLINARY RESEARCH}

\section{The Challenge of Cross-disciplinary Research}

Cross-disciplinary knowledge is called for because real-world problems, such as water management, do not come in disciplinary-shaped boxes (Jeffrey 2003). A broad range of competencies is required to deal with these technically and socially complex issues. Putting together a good cross-disciplinary research proposal is not an easy task, however, and according to Sperber (2006), it can often result in a kind of "cosmetic interdisciplinarity," where the links between disciplines remain very superficial. In general, problems become apparent during project implementation when communication between disciplines is essential to achieve joint products. According to Bruce et al. (2004: 458), who studied interdisciplinary projects in the European Fifth Framework program, the need for interdisciplinary research, especially between natural and social sciences, is not met by the research community and "few studies [are] available on which to base policy recommendations for the support and management of interdisciplinary research." Cross-disciplinary research thus remains a very challenging endeavor.

Scientific disciplines distinguish themselves through different areas of interest, assumptions, priorities, vocabularies, methods, research practices, and communication media (associations, journals, conferences). These elements work together to constitute professional communities at the level of disciplines or sub-disciplines, into which researchers are socialized. In scientific organizations such as universities, which are traditionally structured according to disciplines, the latter can have strong effects on professional and even personal identity.

A number of different terms have been proposed to distinguish between levels of working beyond the borders of one's own discipline: multidisciplinarity, inter-disciplinarity and transdisciplinarity. For more background on these varieties see Bruce et al. (2004), Stokols et al. (2003) and Lawrence and Després (2004). We use crossdisciplinary research here as a more general and descriptive term embracing all meanings referred to above. One goal of our analyses is to find out how to facilitate cross-disciplinary collaborations aimed at integrating knowledge from different backgrounds.

\section{Framing}

In this paper, we analyze differences and collaboration between researchers from different disciplines from a framing perspective. The process of framing has been studied in such fields as environmental conflict (Lewicki et al. 2003), decision making (Tversky and Kahneman 1981), and negotiation (Putnam and Holmer 1992). A common denominator in the diverse uses of the frame concept seems to be that something, like a vague notion of a problem, an interaction situation, or a specific set of problem elements, can be understood in different ways, according to different frames, and that this holds different implications for what that something will be taken to mean. A frame can thus be considered a sense-making device (Weick 1995), adding meaning to a previously confusing or less meaningful situation or domain. When people from different backgrounds work together, they tend to frame the issues at hand in very different ways by defining differently "what this is all about." 
The contexts where framing is relevant are frequently characterized by the active construction of meaning among multiple actors in "emergent organizational contexts" (Bouwen 1998), where common sense has to be made out of confusing or ambiguous situations. We adopt a discursive approach to framing (Dewulf et al. 2004), by focusing on how people define the meaning of an issue or how they negotiate the proper frame through the way they use language in their interactions with each other.

\section{Framing in Cross-disciplinary Research}

Cross-disciplinary research constitutes a context where multiple ways of framing the issues are likely. Each scientific (sub-)discipline orients its attention to certain phenomena, and takes a specific approach to conceptualize and study these phenomena. Each discipline thus maps a specific area, and maps it in a specific way (highlighting specific features of the area, using certain kinds of symbols, etc.). As Judge (1995) argues, these maps can be very diverse, overlapping, and difficult to reconcile, and yet everyone does not need the same map. The selectivity of a specific theoretical perspective or methodological procedure is what allows (sub-) disciplines to become very good at understanding a particular kind of phenomenon from a particular point of view. However, when a research project is set up where people from different disciplines work together, it is unlikely that these different orientations, methods, and conceptualizations will easily fit together.

Disciplinary background seems likely to influence how researchers make sense of a common issue. In a study of an interdisciplinary network on human impacts on ecosystems, Westley et al. (2003) found that a major problem was problem definition. It played a key role in the entry stage of the interdisciplinary collaboration, revolving around such questions as: who defines the nature of the problem, the scale of analysis (genetic, landscape, ecosystem), or the level of complexity (deterministic, stochastic, or chaotic)?

From a framing perspective, one can expect that researchers in cross-disciplinary collaboration will differ in the way they draw boundaries around an issue by including or excluding certain issue elements (different boundaries); in the issue element (s) they put into the focus of attention (different central concepts); and in which issue elements they use as encompassing and which they use as constituent elements (different overarching concepts) (Dewulf 2006). In this study, we focus on differences in issue framing in order to capture diversity at the level where it takes the form of divergent views on the issues to be researched.

The specific challenge posed by the confrontation of diverse frames of reference can be understood as ambiguity or the simultaneous presence of multiple ways of understanding a situation (Dewulf et al. 2005). This ambiguity can vary in intensity from a slight indistinctness, through confusion to tension and conflict. When differences in issue framing between disciplines emerge, people start negotiating these boundaries and conceptual arrangements in a process that can range from defending disciplinary positions to the creative construction of new transdisciplinary frames.

In general, dealing with ambiguity or different frames requires not doing away with the differences too quickly but exploring them in a constructive way (Dewulf et al. 2004). Exploring a difference means at the same time valuing and questioning both sides of the difference. This clarifies what the difference consists of, and can provide starting points for connecting the different frames. Bruce et al. (2004: 465) similarly argue that a good interdisciplinary researcher will have a high tolerance for ambiguity, and will refrain from reducing a problem to a limited set of dimensions, but rather take the time to explore the dimensions and boundaries of a problem. Exploring and connecting different ways of framing the issues is not just an intellectual task. The way issues get framed has important relational implications. Questioning our differences in how we frame the issues is a potentially risky activity for the way we relate to each other. A workable relationship has to be found between the different frames and the people using these different frames of reference (Dewulf 2006).

If faced with ambiguity, adding more and more information will likely only increase the ambiguity rather than reduce it. What is needed then are more - and more varied-cues and mechanisms that "enable debate, clarification, and enactment more than simply provide large amounts of data" (Daft and Lengel 1986: 559), in order to create meaning through discussion and joint interpretation. Rich communication media such as meetings and direct contact become more important than poorer 
impersonal media such as formal information systems and special reports (Weick 1995: 99).

Framing has been identified as an important process in social learning (Bouwen and Taillieu 2004). PahlWostl (2002) mentions the following framingrelated aspects as important elements of social learning: construct a shared problem definition among a group of actors; build trust as a basis for critical self-reflection, which implies recognition of different perspectives and how they pertain to decision making; and reflect on assumptions and subjective valuation schemes. These elements also point to the importance of the quality of the interaction processes between people for fostering social learning (Bouwen and Taillieu 2004). For interdisciplinary research specifically, Bruce et al. (2004: 457) stressed the importance of consortium development, team building, and communication.

In the following section, we explain how we use this theoretical approach to study a large-scale crossdisciplinary research project, where we focus on three related research questions:

1. How do researchers differ in framing a central concept?

2. What challenges do framing differences pose for the project?

3. How useful are the interventions undertaken from a social-learning approach for dealing with these differences in framing?

\section{METHODS}

Multiple qualitative research methods were used in this study. With these methods, we do not aim at charting the frequency or intensity of certain phenomena throughout the project, but at better understanding them by studying a theoretically relevant sample (Charmaz 2000). The interviews, field notes, and evaluations were analyzed using the qualitative analysis software "Atlas-ti" (www.atlas ti.com).

Based on interviews with members of the project consortium, we analyzed important differences in the ways adaptive management is framed in the project. We selected a diverse group of eight (of about 100) consortium members. Each interviewee came from a different research institute, and collectively, they represent the broad range of disciplines involved in the project. Half the interviewees are key people involved in the formulation and execution of the project, and the rest play a less central role in the project.

The interviews were conducted by one of the authors, in English, and were audio recorded. The interviewer asked open questions, such as: "What is your interpretation of adaptive management?" "Are you aware of other interpretations in the project?" and "How do these different interpretations affect the project?" These interviews provide a snapshot of some of the relevant frames and frame differences at a certain point in the project, namely at the first general assembly 11 months after the project started. These views may have changed since that time in response to the ongoing discussions in the project.

Because of the importance of language and vocabulary in the approach to framing we outlined above, we took a discourse analytical approach to analyzing the interviews (Wood and Kroger 2000), looking for differences in how the interviewees construct the meaning of adaptive management through the linguistic formulations they deploy (Edwards 1997). To this end, the interviews were fully transcribed. The frame difference dimensions reported in the results section were inductively derived from coding and comparing interviewees' statements related to adaptive management. Given the relatively limited number of interviewees, we do not claim to have identified all relevant frame differences in the project concerning adaptive management, or to have assessed their respective weight in the project. We do claim to have identified, on the basis of a comparative analysis of the 25000 words comprising the text base of the interviews, four important dimensions of difference in the way adaptive management is framed in the project.

The authors also acted as participant observers within the project with respect to the crossdisciplinary process, taking notes during meetings about the ongoing interaction, and video- or audiorecording a number of meetings. These field notes and recordings were used as the basis for answering research questions two and three. Ten project meetings that occurred over the first 18 months of the project served as the basis for analysis. 
Participatory evaluations and reflections were conducted on a number of occasions during or at the end of project meetings. In a feedback round, participants were given the opportunity to voice positive and negative feelings, experiences, or observations they had about the current or past meetings. These participatory evaluations and reflections were also recorded and transcribed, and used as data for research question three.

\section{RESULTS}

The structure of the results section reflects the set of three research questions. The first part analyzes different ways in which the central concept of adaptive management (AM) is framed in the project. The second part analyzes what kind of challenges this diversity of frames poses for the project work. The third part analyzes experiences with interventions in the project (organizing interactive workshops, facilitation, group model building, and using concrete case contexts) aimed at dealing constructively with this diversity of frames. Because of space limitations and the qualitative nature of the data, an important part of the supporting quotes and observations are discussed in Appendices I-III.

\section{Different Ways of Framing Adaptive Management}

From the interviews, we inductively identified four dimensions of difference in how the interviewees frame the central concept of AM: (1) the centrality of learning and experimentation in AM varies; (2) the role of uncertainty is framed differently; (3) AM can be understood primarily as adaptive capacity or as an AM regime; and (4) differences appear in specifying who adapts to what. We will discuss these dimensions one by one here, and illustrate our arguments with a table and quotes from the interviews in Appendix I.

The professional trajectory, in terms of disciplines, of most of the people we interviewed was more heterogeneous than we had expected (see Appendix I). The relation between AM frames and disciplines is, therefore, more complex than a one-to-one correspondence. This makes it hard to tell exactly how the frame differences we found are related to the disciplinary background of the interviewees. However, even between those researchers who have crossed the boundaries of different disciplines, we still found important differences in what AM means for the interviewees.

\section{Learning and experimentation}

Learning is a recurring aspect in the interviewees' statements about AM, but the importance of this learning process for AM is framed in different ways, ranging from central to peripheral. The nature of the learning process is also portrayed differently, specifically with respect to the central vs. limited role of experimentation (hypothesis testing through policy experiments). Finally, the interviewees differ in specifying who should be involved in the learning process.

\section{Uncertainty}

Another aspect in which framings of AM differ concerns the importance of uncertainty. Uncertainty is mentioned variously as an important aspect of AM, a marginal aspect, or not mentioned at all. Those who mention uncertainty do not necessarily mention it in a uniform way. One way of conceiving uncertainty stresses the unpredictability of the system. Another way focuses more on the different views of scientists and stakeholders about some key parameters of a change trajectory.

\section{Adaptive management regime vs. adaptive capacity}

The interviewees variously prefer to talk about AM or about adaptive capacity. The difference here lies in conceiving of AM as (1) a management system with an internal logic and a coherent set of elements (in the sense of "adaptive management regime"); or (2) a dimension that can be applied to management systems of very different kinds (adaptive capacity). The former seeks a general profile of AM systems in terms of coherence between a set of elements, whereas the latter looks for the adaptive merits of specific management systems in specific contexts.

\section{Who adapts to what?}

When interviewees use the terms "adapting," "changing," or "learning," they often specify additional aspects: (1) who or what is adapting, changing, or learning? (2) what is it that they adapt, change, or learn? and (3) in response to what do they adapt, change, or learn? The interviewees specify these aspects in different ways. With regard to all three aspects, both biophysical and social system 
elements are mentioned. The actor responsible for the adaptation (aspect 1, e.g., the people) in one formulation, can become an external factor (aspect 3 , e.g., changing preferences of people) in another formulation. In terms of framing $\mathrm{AM}$, these differences indicate considerable divergence and potential for confusion in defining the boundaries of an AM system, by selecting those aspects that are inside the system (aspects that are adapting or being adapted) and others that are outside the system (aspects that the system adapts to).

In total, we could identify four dimensions of difference in how the concept of AM is framed in the project. Through the way the interviewees include and assemble elements in the way they talk about AM, they construct their understanding of $\mathrm{AM}$, in which certain aspects figure as centrally important, while other aspects are not or are only marginally considered. Given the central place of the AM concept in the project, these differences have implications for project activities. The aspect of how to go about learning and the necessity of policy experiments-an issue that resonates with the AM literature, see, e.g., Lee (1999) - has important implications for conceptualizing the AM cycle, or for the kind of projects that are studied or set up in case studies. Different ways of looking at uncertainty can have implications for the range of water management strategies that are considered. The different assumptions implied by the notions of adaptive capacity vs. AM regime have implications for how the adaptiveness of a water management system is methodologically assessed. The way the question "who adapts what in response to what" gets answered, has important implications in terms of which aspects in a system are considered to be given, which aspects are considered as suitable for intervention, and who should take action. How a central concept is framed can thus have important implications for important project activities, such as the construction of a common conceptual framework, the choice of research of methods or the planning of interventions in the case studies.

\section{Frame Diversity as a Challenge for the Project Work}

Adaptive management is not the only concept that gets framed in different ways. Although we did not study these in detail, other core concepts like vulnerability, resilience, or uncertainty seem to generate a similar kind of ambiguity when researchers from different backgrounds interpret them from different perspectives. The different ways in which these central concepts are framed pose particular challenges to the project, in terms of mutual understanding and coordination. These challenges are illustrated in Appendix II.

1. Very few concepts are self-evident to all participants. It proves very difficult to find a meaningful starting point from which to construct a conceptual framework for the project. The difficulty here lies in finding words that make sense to everybody (even if this sense differs from person to person). Whichever concept is chosen, there are people who are unfamiliar with it or for whom it does not make much sense.

2. Considerable confusion about concepts emerges in project meetings. In some cases, when a meaningful concept is found, it is used by several people but with very different meanings or connotations. In other cases, very different concepts are used to refer to practices or phenomena that are very similar.

3. The different concepts and meanings are not neutral. From their socialization in specific scientific communities, people often feel strongly about which concept to use, especially if this concept is supposed to be used for a joint project task across different organizations or backgrounds. As concepts or ways of framing issues are often linked to specific communities, including or excluding a certain concept can have important implications for the position of people in the project, e.g., who gets a leading role for that part of the project, or who is considered the expert on a certain topic.

\section{Dealing with frame diversity: experiences with interventions}

In this context of frame diversity, integration is a highly needed but at the same time difficult process. Conflicts or the absence of communication can lead to fragmentation, where different frames remain disconnected and different groups continue to work with their own concepts and methods. A slightly 
more desirable but not yet satisfactory outcome would be the dominance of one frame over the others. Opting for integrated computer simulation models, for example, would constrain the type of knowledge that can be included. In particular, the interpretive and qualitative approaches of the social sciences are very difficult to integrate in such an approach, which could lead to joint products but at the expense of excluding certain types of knowledge. The most desirable and yet most challenging approach would be a process of integration that leaves sufficient openness to include a wide range of different frames. It would imply that a diversity of frames can co-exist and be connected without resulting in fragmentation and thus in a collection of disconnected pieces of knowledge. This should be possible in a participatory and interactive process where genuinely new frames may be developed and explored. In the following section, we focus on a number of interventions that have been carried out in the project in order to stimulate the exploration, connection, and integration of different frames.

In the absence of clear guidelines or established practices for cross-disciplinary work, a number of experiments in social learning (Pahl-Wostl 2002, Bouwen and Taillieu 2004) are being tried out in the project, to deal with the ambiguities provoked by the divergent frames and to foster constructive ways of connecting knowledge from different backgrounds (Pahl-Wostl 2006). The methods employed draw on the fields of participatory stakeholder processes, integrated assessment and management, multi-actor collaboration and organizational development. We discuss and evaluate these attempts in the following subsections.

\section{Organizing interactive workshops}

A general approach in the studied project is to organize interactive workshops to exchange ideas during face-to-face discussions, to develop a common language and a basis for understanding, and to decide on courses of action. As we argued above, connecting frames is a challenge on both the content and the relational levels. Content can be transmitted through other means of communication as well, but relational connecting is much more powerful in face-to-face interaction than through more impersonal means of communication.
From the participants' evaluations and our observations, which are documented in Appendix III, we can conclude that the participants generally valued the workshops, and that they especially valued the more interactive parts of the workshops, like working in small break-out groups. The latter were evaluated as more productive and contributing to a good atmosphere among the participants. Open and mutual questioning, an important aspect of exploring different frames, seemed to become possible in these workshops, especially in the smaller and more interactive meeting environments. As a downside, participants mentioned the high investment of time and resources that workshops require, and the problem of the ever-changing constellation of people at workshops-this can considerably slow down progress in relational connecting and learning to work as a group.

\section{Facilitation}

In the interactive workshops, the session format was mostly presentation followed by a question and answer session. This generally does not stimulate a lot of discussion. It is not easy for presenters to take up the double role of presenting a lot of content and chairing the meeting. Presenting calls for clear explanation and information flow from the presenter to the audience. Chairing the meeting calls for stimulating participation, checking comprehension, following up on comments, and explicating (and checking) the goal of the meeting and what we expect as outputs. It is not an easy job to combine both these roles in one person, and presenters seemed to have difficulties with it, resulting in situations where the frame of the presenter dominates the meeting.

Some of the interactive workshops were facilitated by qualified project members. In general, the complementary roles of presenting vs. facilitating worked well. The presenter can concentrate on the subject, while the facilitator is in touch with the audience, prepares the upcoming interaction, and invites and structures the discussion. This proves very comforting for the presenter. However, some people succeed in fulfilling both roles, by combining their scientific understanding with process skills. Even in an informal setting, they can thus obtain high credibility and acceptance by the group. We document this in Appendix III. 


\section{Group model building}

The project coordination team decided to adopt a participatory model building process supported by a binding yet flexible graphical notation, namely Unified Modeling Language (UML). This would support the construction and understanding of a common conceptual framework, in which graphical representations are combined with narratives that document the line of argument.

From participant evaluations and our observations, which are documented in Appendix III, we can draw the following conclusions. Using UML in group discussions helps make mutual assumptions explicit, because everybody attempts to translate his or her concepts into a common language. In selecting aspects, labeling them, drawing the relations and labeling the relations, differences between participants' frames can emerge and can be discussed. Creating the diagrams also helps identify where knowledge of a system or process is incomplete. An advantage of UML is that it does not rely on the often implicit and possibly diverging meanings of the visual aspects of the diagram. The disadvantage is that people may still read those meanings into the visual aspects of the diagram and make diverging conclusions.

Working with UML as a tool for documenting and exchanging knowledge can be interpreted with Wenger's (1998) participation-reification concepts. Participation, meaning here involvement in the development of knowledge, needs reification, e.g., diagrams (or papers or reports) to store and "transport" this knowledge. But reifications always need participation: people need to be willing to learn and to use the new language and to work with it, otherwise the diagrams remain meaningless. This learning process will have to be partially redone every time a broader group is expected to work with the new language.

\section{Using concrete case contexts}

Using concrete case contexts in an interdisciplinary environment can be done for a number of reasons. Explaining or illustrating theoretical points with examples from a jointly available case context makes them more understandable for others, and can make explicit important differences in understanding.
In order to deal with different frames, some kind of anchor point is needed that allows working constructively with the diversity. Concrete case contexts can provide this necessary common ground when different theoretical approaches have to be dealt with, because they provide a kind of anchor point for keeping the discussion focused and the exploration of different views going. This requires that the specific case that is used to focus the discussion is sufficiently known by the different participants.

As we illustrate with observations and participant evaluations in Appendix III, using concrete case contexts was found to be motivating and helpful for clarifying concepts. When the level of detail and complexity of a case situation is too high to work with, simplified or stylized representations of case situations were used with apparently positive results. Asking researchers from different backgrounds to apply their respective concepts and methods to a concrete case description that is available to all participants, was evaluated as helpful for eliciting and understanding the different frames of reference.

Schön and Rein (1994) have similarly argued that "situated" frame reflection is needed for dealing with frame conflicts in policy controversies. They claim that "when policy controversies are abstracted from the situations in which they arise, as in academic discourse, they are removed from the pace and pressure of the policy arena, but they exist in a kind of vacuum where it is hard to imagine how they might ever be resolved" (Schön \& Rein 1994: 176). As a strategy for resolution, they propose reflecting on the involved frames with the aim of getting to a pragmatic solution in a specific context. Therefore, the role of the seven case studies in the project could be crucial in fostering cross-disciplinary outcomes.

\section{DISCUSSION AND CONCLUSIONS}

We started by identifying the necessity of crossdisciplinary research collaboration for achieving a better understanding of how human and natural systems are dynamically linked. Single disciplines are generally ill-equipped to deal with issues that are both technically and socially complex and interdependent. On the other hand, genuinely crossdisciplinary research appears difficult to put into practice. 
We analyzed cross-disciplinary research collaboration from a framing approach, focusing on the different frames of reference that researchers use to make sense of the issues that they want to study jointly. From analyzing interviews with members of a largescale research consortium on adaptive water management, we inductively identified four dimensions of difference in the way the researchers framed the central concept of AM: (1) they framed the centrality of learning and experimentation to AM in different ways; (2) they framed the role of uncertainty differently, in terms of unpredictability or in terms of different views; (3) they framed AM either as a management system with a coherent set of elements ("regime") or as a dimension that can be applied to management systems of very different kinds ("adaptive capacity"); (4) in specifying "who is adapting?" "what is that they adapt?" and "in response to what do they adapt?" the interviewees' answers diverged significantly.

Some of the challenges of cross-disciplinary research could thus be better understood as dealing with the ambiguity and tension provoked by the simultaneous presence of multiple ways of framing or understanding a situation or issue (Dewulf et al. 2005). By analyzing meeting observations, it appeared that very few concepts are self-evident or neutral for all project participants. Considerable confusion about concepts emerges in project meetings because either the same concept is used by several people but with very different meanings, or conversely, very different concepts are used to refer to practices or phenomena that are very similar. A cross-disciplinary research approach calls for integration of different kinds of knowledge into a new framework but the aforementioned challenges indicate that this is not a straightforward process.

A number of interventions were tried out in the project from a social learning approach (Pahl-Wostl 2002, Bouwen and Taillieu 2004), in order to foster constructive ways of connecting knowledge from different backgrounds. It is too early to make final judgments about whether the approaches chosen will be successful, or if the considerable investment of time by project participants in joint activities will result in innovative products that would not have been possible without this investment. Nevertheless, on the basis of participants' evaluations and our own observations, we tried to assess the impact of four types of interventions during the first 18 months of the project.
- The participants generally valued the workshops, and especially the more interactive parts, e.g., working in small breakout groups, which were evaluated as more productive and contributing to a good atmosphere among the participants. They allowed for open and mutual questioning, an important aspect of exploring different frames.

- Some of the interactive workshops were specifically designed and facilitated by qualified project members. In general, the complementary roles of presenting vs. facilitating worked well and allowed for more opportunities for open discussion across different frames.

- Using participatory model building in UML helped make mutual assumptions explicit and the differences between participants' frames more visible and understandable.

- Using concrete or stylized case situations as a way to deal with diverse methods or theories, allowed participants to use the case situations as a common ground to which the various frames could be connected.

As we have argued, cross-disciplinary research requires dealing with diverse frames, which often take the form of tacit understandings about how disciplines or theories select, focus, and embed aspects of the world, and how they articulate these issues in a specific vocabulary. If we try to reason a step further from these findings and similar ones (e.g., Dewulf 2006), we can suggest a hypothetical process of optimal steps in dealing with crossdisciplinary frame differences.

1. Get to know each other's frames. A first step is to be confronted with the different kinds of knowledge others contribute.

2. Acknowledge differences. This requires paying attention to differences and not acting as if there were none.

3. Incorporate other concepts into your own framing. A first and perhaps inevitable step in understanding other frames is to translate 
them into your own terms. This does not do justice to the full richness of the knowledge, but is probably necessary as first approximation (just as translating words is often a necessary intermediary step when learning a foreign language).

4. Explore and work with the differences. A further step is to mutually explore the different views so that each can understand the other's view in its own terms, and thus find out where the frames are incompatible and where they provide complementary contributions.

5. Forge new frames. As a way of integrating different frames, often a new vocabulary has to be created that is able to carry the new and jointly created meanings and knowledge.

As was evident from the results reported above, these kinds of processes set high requirements in terms of interaction and learning between researchers. Further research is needed to assess whether this hypothetical process leads to the expected results in terms of frame connection and integration, and whether the interventions we reviewed can be fine-tuned to facilitate this process specifically.

Responses to this article can be read online at: http://www.ecologyandsociety.org/vol12/iss2/art14/responses/

\section{Acknowledgments:}

The research for this article was executed as part of the NeWater project (Contract no 511179, 6th EU Framework Programme). The authors would like to thank the European Commission for the financial support received and the NeWater consortium members for their kind collaboration.

\section{LITERATURE CITED}

Bouwen, R. 1998. Relational construction of meaning in emerging organizational contexts. European Journal of Work and Organizational Psychology 7(3):299-319.
Bouwen, R., and T. Taillieu. 2004. Multi-party collaboration as social learning for interdependence: developing relational knowing for sustainable natural resource management. Journal of Community \& Applied Social Psychology 14 (3):137-153.

Bruce, A., C. Lyall, J. Tait, and R. Williams. 2004. Interdisciplinary integration in Europe: the case of the Fifth Framework programme. Futures 36:457470.

Charmaz, K. 2000. Grounded theory: objectivist and constructivist methods. Pages 509-535 in N. K. Denznin and Y. S. Lincoln, editors. Handbook of qualitative research. Second edition. Sage Publications, London, UK.

Daft, R. L., and R. H. Lengel. 1986. Organizational information requirements, media, richness and structural design. Management Science 32:554571.

Dewulf, A. 2006. Issue framing in multi-actor contexts: how people make sense of issues through negotiating meaning, enacting discourse and doing differences. Dissertation. Faculty of Psychology and Educational Sciences, Katholieke Universiteit Leuven, Belgium.

Dewulf, A., M. Craps, R. Bouwen, T. Taillieu, and C. Pahl-Wostl. 2005. Integrated management of natural resources: dealing with ambiguous issues, multiple actors and diverging frames. Water Science and Technology 52(6):115-124.

Dewulf, A., M. Craps, and G. Dercon. 2004. How issues get framed and reframed when different communities meet. A multi-level analysis of a collaborative soil conservation initiative in the Ecuadorian Andes. Journal of Community and Applied Social Psychology 14:177-192.

Edwards, D. 1997. Discourse and cognition. Sage Publications, London, UK.

Gray, B. 1989. Collaborating. Finding common ground for multiparty problems. Jossey-Bass, San Francisco, California, USA.

Jeffrey, P. 2003. Smoothing the Waters: Observations on the Process of Cross-Disciplinary Research Collaboration. Social Studies of Science 33 (4):539-562. 
Judge, A. 1995. Transdisciplinarity through structured dialogue. Knowledge Organization 22:82-88.

Lawrence, R. J., and C. Després. 2004. Futures of transdisciplinarity. Futures 36(4):397-405.

Lee, K. N. 1999. Appraising adaptive management. Conservation Ecology 3(2): 3. [online] URL: http:/ /www.consecol.org/vol3/iss2/art3/.

Lewicki, R., Gray, B., and Elliott, M. 2003. Making sense of intractable environmental conflict: concepts and cases. Island Press, Washington, D. C., USA.

Pahl-Wostl, C. 2002. Towards sustainability in the water sector: the importance of human actors and processes of social learning. Aquatic Sciences 64:394-411.

Pahl-Wostl, C. 2006. The importance of social learning in restoring the multifunctionality of rivers and floodplains. Ecology and Society 11(1): 10. [online] URL: http://www.ecologyandsociety.org/vol11/ iss1/art10/.

Putnam, L. L., and Holmer, M. 1992.Framing, reframing and issue development. Pages 128-155 in L. Putnam and M. Roloff, editors. Communication and negotiation. Sage Publications, London, UK.

Scholz, R. W., D. J. Lang, A. Wiek, A. Walter, and M. Stauffacher. 2005. Transdisciplinary case studies as a means of sustainability learning: historical framework and theory. EHT-UNS Working paper No. 43, ETH Zürich, Umweltnaturund Umweltsozialwissenschaften, Zürich, Switzerland.

Schön, D., and M. Rein. 1994. Frame reflection: toward the resolution of intractable policy controversies. Basic Books, New York, New York, USA.

Shotter, J. 2004. Expressing and legitimating "actionable knowledge" from within "the moment of acting." Concepts and Transformation 9(2):205229.

Sperber, D. 2006. Why rethink interdisciplinarity? Interdisciplines [online] URL: http://www.interdisc iplines.org/interdisciplinarity/papers/1.
Stokols, D., J. Fuqua, J. Gress, R. Harvey, K. Phillips, L. Baezconde-Garbanati, J. Unger, P. Palmer, M. Clark, S. Colby, G. Morgan, and W. Trochim. 2003. Evaluating transdisciplinary science. Nicotine and Tobacco Research 5 (Suppl. 1):21-39.

Tversky,A., and D. Kahneman. 1981. The framing of decisions and the psychology of choice. Science 211:453-458.

Wenger, E. 1998. Communities of practice: learning, meaning and identity. University Press, Cambridge, UK.

Weick, K. 1995. Sensemaking in organizations. Sage Publications, Thousand Oaks, California, USA.

Westley, F., F. Miller, and B. Lacy. 2003. Far from land: further explorations in consilience. Paper presented at the Tenth International Conference on Multi-Organizational Partnerships, Alliances and Networks, "Co-creating Emergent Insight". 25-28 June 2003, University of Strathclyde, Glasgow, Scotland.

Wood, L., and R. Kroger. 2000. Doing discourse analysis: methods for studying action in talk and text. Sage Publications, Thousand Oaks, California, USA. 
APPENDIX 1. Different ways of framing "adaptive management": quotes from the interviews

In this appendix we present quotes from the interviews on adaptive management (AM) to illustrate and support the four dimensions of frame difference we discussed in the main text. We use I1 to I8 to refer to the eight interviewees.

When we asked interviewees which scientific discipline they belong to most interviewees were not able or didn't like to label themselves in terms of one specific discipline (I1, I2, I3, I5, I6). They described different disciplines they had been working in over the course of their career or they mentioned a field that is interdisciplinary in itself (e.g. integrated assessment, management sciences, integrated water management). Some of them (I2, I6) framed their background as consisting of an initial field of education and several fields of interests. Although most interviewees had a background characterized by multiple disciplines they can hardly be considered generalists. There still was a clear difference in background and in the focus of their research.

Text analysis of the interviews led us to the identification of four dimensions of difference in the way the interviewees frame AM. These dimensions are presented with illustrative quotes in the following table and then discussed one by one. 


\begin{tabular}{llll}
\hline \hline $\begin{array}{l}\text { Learning and } \\
\text { experimentation }\end{array}$ & Uncertainty & Adaptive capacity Who adapts to what? & $\begin{array}{l}\text { Disciplinary bac- } \\
\text { kground }\end{array}$ \\
\hline
\end{tabular}

I1

"AM is learning to
manage by managing to
learn"

"manage complex

systems in an uncertain world by simply being able to adapt to new insights" "a system" / "people" <adapt> "management decisions" <in response to> "new insights" / "changing management objectives"

"academics" / "policy makers" / "people in business" / "people without any training"

$<$ learn>

"together"

$<$ in response to $>$

"failure to learn" / " catastrophes"
I3 "I deeply believe that, the social change dimension of AM ... can be reached only through experimentation" uncertainty with respect to (1)

"where we are" (2)

"where we want to go", (3) "which path to follow" and (4) "monitoring" "social system"

<adapts>

(unspecified)

<in response to>

"change"
Chemistry; Molecular biology; Environmental Physics; Integrated assessment; Social sciences

Systems Ecology; Cognitive Psychology

Computer Science; Management Sciences

Agricultural Engineering; Hydrology ... is actually the most important goal, the most important thing uncertainty you have an adaptive capacity which is able to accommodate surprises" at"

"stakeholders organized as a coordinated group" / "the system" <adapt> (unspecified)

<in response to>

"a structural change in external conditions" / "changing of the preferences of your people"

I5 "definitely learning and learning together"

"the learning aspect, where you explicitly try to engage in experiments and learn from that"

"you want to deal with uncertainty ... you want to prepare yourself for different futures" "you want to strengthen the adaptive capacity of the system" "both ecosystems and actors or people" / "scientists together with stakeholders"

<adapt>

"water management strategies"

"river flow"

<in response to>

"enlarged scope of situations

that might happen to you" /

"change"
Water Resources Engineering and Management; Environmental mental Physics 
I6

"It is a process of exercising, and act then learn, then learn some more, then act again"

I7

"It basically adds a sort of a learning aspect, a learning component to IWRM" "create some sort of responsiveness within your system to react in a better way to things that might happen in the future"

\author{
"The events they, \\ you can't predict \\ them. And you also \\ don't know how \\ the frequency and \\ the magnitude of \\ this event are \\ developing. And so \\ you have to create \\ a management \\ system that is able \\ to react to these \\ events"
}

$\begin{array}{ll}\text { "we need to do "a hundred people together } \\ \text { that by increasing } & \text { across two dozen stakeholders" } \\ \text { the ability to } & \text { <change> } \\ \text { adapt, so adaptive the system } \\ \text { capacity comes cin response to> } \\ \text { into the language "unfolding risks as they occur" } \\ \text { quite quickly" }\end{array}$

Political ecology; Social geography the ability to <change> adapt, so adaptive the system into the language "unfolding risks as they occur" quite quickly"

\author{
"the stakeholders" \\ <adapt> \\ "strategies or actions" \\ $<$ in response to> \\ "scenario's" / "things that might \\ happen" / "issues challenging \\ our management"
}

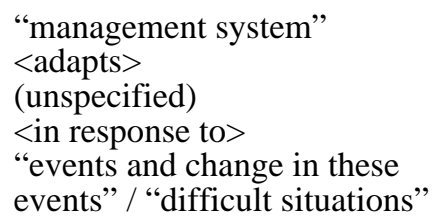

Political sciences; Social sciences

Management Sciences

\section{Learning and experimentation}

Learning is a recurring aspect in the interviewee's statements about adaptive management (AM). For one interviewee (I7) the learning cycle is what sets AM apart from IWRM, and thus a key aspect in defining AM. Four others (I1, I2, I5, I6) also mention learning as a key element of AM: "AM is learning to manage by managing to learn" (I1); "AM to me has a lot to do with learning, because it was developed in response to failure to learn" (I2); "definitely learning and learning together" (I5); "It is a process of exercising, and act then learn, then learn some more, then act again" (I6). Three interviewees do not refer to learning in their statements about AM.

Interviewees differ in the extent to which they conceive this learning process as consisting mainly of hypothesis testing through policy experiments. Five interviewees mention experimentation, and for two of them, learning pretty much means experimentation: "the learning aspect, where you explicitly try to engage in experiments and learn from that" (I5); "it is a cycle of learning, assessing a problem, then posing hypotheses ... the policy now is the test of your best hypotheses" (I2). The other two (I1, I7) mention experimentation as an additional possibility but a very crucial or viable way of learning in AM. Interestingly, one interviewee (I6) mentions learning but not experimentation, and another (I3) mentions experimentation without learning, indicating again that learning and experimentation are not used as synonyms among the interviewed researchers.

Interviewees differ also in whether they specify the actors of the learning process, or who should be involved in the learning process: e.g. mainly the responsible water managers; or the whole group of scientists, policy makers and stakeholders; or scientists and stakeholders. 


\section{Uncertainty}

Two of the eight interviewees do not refer to uncertainty when asked about their definition of AM. Of these six, one mentions uncertainty only as a marginal aspect (I7), and the rest do include uncertainty as an important aspect of what AM means. However, they do not necessarily mention it in a uniform way.

The interviewees mentioning uncertainty as an important aspect of what AM means, mention it in different ways. The following interviewees stress the unpredictability of the system as follows:

- I1 links uncertainty to the complexity of the systems and the limits to predictability.

- I4 links uncertainty to stochastic drivers of the system, which generate surprises.

- I8 links uncertainty to the unpredictability of events, their frequency and magnitude.

- I5 links uncertainty to different possible futures.

In addition I3 identifies uncertainty with respect to (1) where we are, (2) where we want to go, (3) which path to follow, (4) monitoring. He focuses on uncertainty as a consequence of different views between scientists and/or stakeholders about some key parameters of a change trajectory.

\section{Adaptive management regime versus adaptive capacity}

Although the expression used in the interview questions was "adaptive management", three interviewees (I4, I5, I6) draw strongly on the concept of 'adaptive capacity' for explaining their views. Only one interviewee spontaneously uses the term regime while explaining adaptive management.

Two interviewees (I4, I6) claim the term adaptive capacity to be better suited for the project. I4 calls it "the most important goal, the most important thing that you should look at". I6 stresses adaptive capacity as "the ability to adapt" and contrasts this with the notion of a 'regime', because "that seems to imply ... all sorts of assumptions about institutions and so". He argues, for example, that "you can have very effective adaptive management regimes that are completely hierarchical or oligarchical".

\section{Who adapts to what?}

When interviewees use the terms 'adapting', 'changing' or 'learning', they often specify additional aspects: (1) who or what is adapting, changing or learning?; (2) what is it that they adapt, change or learn; and (3) in response to what do they adapt, change or learn? The interviewees specify these aspects in different ways. In the above table, we represented these three elements per interviewee, and combined them with the specific terms that the interviewee uses as operators (between $<>$ ).

(1) Often the first question is left unanswered in how they talk about adapting, but when specified, the following kinds of things are said to be adapting, changing or learning: 'the system', 'the people', 'the ecosystem', 'the stakeholders', 'scientists and stakeholders' or 'the management system'.

(2) The second question is also left unanswered in many cases, but 'management strategies', 'management decisions', 'river flow' and 'the system' are mentioned as things that are adapted or changed.

(3) With respect to the third aspect, the following things were mentioned in response to which adaptation, change or learning occurs: 'change', 'changing management objectives', 'new insights', 'structural change in external conditions', 'new external situation', 'external change', 'changing preferences of the people', and 'flood disasters'.

Interestingly, with regard to all three aspects both biophysical ánd social system elements are mentioned - the general 'system' mostly stands for both. Starting from the following questions: (1) who 
or what is adapting, changing or learning?; (2) what is it that they adapt, change or learn; and (3) in response to what do they adapt, change or learn?; we can try to identify the possibilities by structuring the three aspects into either biophysical or social system elements. Thereby we get the following eight combinations:

1. the biophysical system adapts the biophysical system in response to biophysical changes (e.g. complex adaptive ecosystems under climate change)

2. the biophysical systems adapts the biophysical system in response to social changes (e.g. complex adaptive ecosystems under human-induced stress)

3. the social system adapts the social system in response to biophysical changes (e.g. learning to live with water)

4. the social system adapts the social system in response to social changes (e.g. the government starts subsidizing drinking water service for single parent families)

5. the social system adapts the biophysical systems in response biophysical changes (e.g. creating floodplains in response to extreme events)

6. the social system adapts the biophysical system in response social changes (e.g. making polders in response to need for arable land)

7. the biophysical system adapts the social system in response to biophysical changes (e.g. replacement of species due to climate change which may result in long-term trends in water availability)

8. the biophysical system adapts the social system in response to social changes (this seems logically impossible)

This list serves as a thought experiment about possible meanings of adaptation. The broad range of possibilities does not even take into account that parts of a subsystem can change other parts of the same subsystem (e.g. one part of the social system can change another part of the social system). This illustrates how adaptation can be understood in very diverging ways. 
APPENDIX 2. Frame diversity as a challenge for the project work: observations

On many occasions researchers in the project were confronted with the different ways concepts are framed. In trying to understand new concepts, or concepts they are not familiar with, people interpret them differently. These differences are often based on their disciplinary background and the research tradition they are connected with. The result is considerable confusion in project meetings, which makes mutual understanding and collaboration difficult.

This challenge has been expressed by many researchers in the project with comments as:

- "In the project we have very little shared understanding."

- "How can we get beyond the jargon?"

An important number of concepts that were used in the project proposal and at the initial project meetings were unfamiliar for a part of the project members. A participant in the project kick-off meeting, for example, reported that she was unfamiliar with the "mental model" concept, which was used by participants with a psychological background, while others didn't get the meaning of "code" used in the sense of a piece of software programming by participants with a background in computer modeling.

Often the same concept was used by several people but with very different meanings or connotations, as we illustrated in our analysis of adaptive management. Another good example is the 'regime' concept, mainly used in the sense of a water management regime. However, for the political scientists in the project, 'regime' and certainly 'regime change' means something very different, and for people with a water management background 'regime' can also refer to the flow regime of a river.

On the other hand different concepts are used to refer to very similar practices or phenomena. An example of this are the various concepts used to refer to interactively constructing a conceptual modela method that has been used and adapted in different disciplines and is variously referred to as 'causal loop diagramming', 'group model building', 'causal mapping' or 'Bayesian network analysis'.

Hereafter, we illustrate in more detail how different ways of framing a concept played out in two episodes from two different project meetings.

\section{Episode 1. Protocols for vulnerability assessment}

In one of the workshops different protocols were offered to assess vulnerability in the cases. The protocol that was presented on the first day was based on work in the social sciences. It was an exercise in collecting qualitative data, defining boundaries of exposure units and rating vulnerability based on different kinds of criteria. At the end of that day some participants looked very worried. One of the case study leaders said not to feel capable of bringing this approach to the stakeholders. Most participants were from a quantitative modeling background and had difficulties in working with qualitative data and in assessing their validity. This piece of conversation, reconstructed from meeting notes, can illustrate this kind of challenge. At the end of this sequence one participant frames filling in a table with qualitative data as a 'game', while the presenter stresses that not the narrative in itself is important, but the analysis is.

- Presenter: "Collect the narrative of the basin, no analytical data but the story. Go back to the history of the basin."

- Participant: "Who can tell which data are really necessary?” 
- Presenter: "Rather qualitative data"

- Other participant: "I need guidance to fill in the table, I hate games."

- Presenter: "The narrative without analysis is inappropriate."

Those unfamiliar with qualitative research methods struggled also with the following aspects. Defining the boundaries of the river basin and of exposure units was considered very arbitrary and thus biased. They became aware that the vulnerability table is filled in from one particular perspective. And the way vulnerability was rated, was called subjective. The presenter's comment that "making categories is always a kind of judgment" didn't really comfort them. They truly wondered how one can deal with the 'bias' caused by one's own perspective.

\section{Episode 2. Bayesian network tool}

In the course of a meeting the 'Bayesian network tool' was presented as a participatory tool. Social scientists involved in participatory processes questioned the presenter: "What is your network consisting of? Is it a social network?" It was clarified that the 'network' concept did not refer to a network of people (as is often assumed in social science) but was referring to the elements of the model and the cause-effect relationships among them. The role of the stakeholders also became clearer. They were involved in the modeling process to bring in local knowledge about the system to be modeled and individual or group perspectives.

Presenter: "Stakeholders bring their values and views to the network."

Participant: "What happens when their views differ? Do you start a process to create something in common or do they continue to exist as different views?"

Presenter: "Yes, that's life. The Bayesian network shows what the consequences are if you take one perspective and then when you take the other one."

The qualification 'participatory' didn't refer to stakeholders acting in a negotiation process to connect different perspectives (as some of the participants were assuming), but rather to involving stakeholders as a source of information. 
APPENDIX 3. Dealing with frame diversity: evaluations and observations

Four different kinds of interventions in the project have been observed from a social learning perspective. We used participant evaluations and our own observations during these workshops to assess the impact of these four kinds of intervention. How do they enable to deal with the ambiguity provoked by frame diversity? And do they foster constructive ways to connect knowledge from different backgrounds?

\section{Organizing interactive workshops: evaluations and observations}

To assess the impact of organizing interactive workshops, rather than relying on written communication, we have assembled evaluations and observations from 6 project workshops. We report the results thematically hereafter.

Participants in interactive workshops valued them for several reasons:

- "People could see each other face to face"

- "Communication and work in the break-out groups, and small internal meetings"

- "We have communications that would not be possible through e-mail, e.g. integration"

- "People got to know what they can expect from each other"

- "People recognized where they fit in, they became aware of linkages"

- $\quad$ "We have tangible and intangible outcomes, like working with the group, which we will realize later"

In this sense, the workshops contributed to the relational side of connecting the people using the different frames: getting to know each other and mutual expectations, finding a place and a role in the project, starting to work as a group.

That the participants referred to the more interactive parts of the workshops as the more fruitful ones adds to this picture. Working in smaller groups was experienced as the most helpful to explore and define new concepts.

- "Break-out groups were the most interesting and useful discussions, the most interactive"

- "We need longer slots for small productive groups"

- "Smaller groups were really positive, working on a clear product keeps you focused"

- $\quad$ "In the discussion we were questioning a lot of things, ..., we were questioning each point we elaborated before, generating new ideas"

- "Products were better because of the small groups, I had fun moments in the break-out groups" Also working with an open format ("market") to stimulate intensive interactions was valued:

- $\quad$ "At the end of the market, I felt that something started to happen, I didn't want to get back to the plenary"

From our observations it was also clear that small group activities during the workshops generated much more lively and in depth discussions than the usual presentation plus questions format. 
According to the experiences of the participants, open communication contributes to the value of interactive workshops.

- $\quad$ "I experienced as positive that everybody is very willing to listen to each others views and opinions"

- "Very good atmosphere, trial and error approach without feeling bad about it is very constructive"

One of the participants reported that his way of framing adaptive systems was changed through a workshop ("my mental model about adaptive systems evolved"). From our observations, an interactive discussion episode during a workshop about the difference between current water management, integrated water resources management and AM allowed an exploration and redefinition of these concepts among the participants in ways that would be very hard to achieve through other means.

As a downside, frustration was also expressed with respect to the high investment of time and resources that workshops require. It seems like there is never enough time in a workshop:

- "It is not possible in one meeting"

- "There was not so much time as I expected"

Furthermore, the important relational effects a workshop can have, impacts only the people who were there, and the results that are generated depend on the group of people that went through that process together.

- $\quad$ "At the same time I feel a bit uncomfortable about randomness: the composition of the group does matter in what we have as result, we should be very conscious about it"

- "I had a low moment this afternoon, because it seems like every meeting is starting anew"

- In this vein, a number of participants experienced as problematic that "people come in and jump out" or are "concerned about earlier leaving".

\section{Facilitation: evaluations and observations}

Evaluations and observations from 4 project workshops served as a basis for assessing the impact of facilitation.

At the end of the kick-off meeting of the project, the difficulty of taking the double role of presenting a lot of information and at the same time facilitating the discussion was identified in a debriefing between the organizers and observers of the meeting. Presenting calls for clear explanation and information flow from presenter to public. Facilitating the meeting calls for stimulating participation, checking comprehension, following up on comments and explicating (and checking) the goal of the meeting and what we expect as outputs. It's not an easy job to combine both these roles in one person, and presenters seemed to have difficulties with it.

This was one of the reasons why a next workshop was specifically designed and facilitated for exploring the different frames of reference of the participants and dealing with them in constructive ways, in order to reduce confusion and increase mutual understanding. This took the form of inviting participants to prepare a 2-slide presentation on their view of the workshop's central topic. These presentations were then given at the start-up of the workshop. In this way, a number of differences in conceptions came to the fore, which might otherwise have remained implicit. The way this phase of the workshop was designed and facilitated allowed people to better understand the background of the others around the table and use that knowledge throughout the further discussions. Comments of the participants when evaluating the workshop show they valued the influence of the facilitators on the design of the workshop 
as well as the role they took during the workshop.

- $\quad$ "The moderation was very good, everyone was able to look beyond one's own perspective in the open break-out groups. We can now position ourselves better."

- "The complementary roles of presenting versus facilitating worked well."

By contrast, in another workshop where only the presenters' role was taken and important differences in interpretations and views between the presenter and the other participants emerged but remained unaddressed, the following comment was given at the end of the day: "Did we have to meet for this ? We could have read this. I expected more guidance."

Sometimes one person is able to fulfill both the roles of participating and facilitating. In that case process skills are combined with a good scientific understanding, the latter increasing the credibility of the facilitator. This situation was present in a workshop that was organized to change the dynamic of a rather polarized discussion between two research groups. A third group of researchers was brought in. They had their own alternative approach and thus contributed to the discussion at the content level. At the same time interventions on the procedural level were made by this third party, leading to shared action plans and thus improving the interaction process. The example shows that the facilitator's role can be taken in an informal way if this is accepted by the group. One of the group members confirmed the effectiveness of this setting: "So in the meeting yesterday we brought in X, who hadn't been part to that, I mean, been part of some of the discussions. A new one can and could immediately see how it could work, could see a role for themselves. The rest of us were willing to allow this."

\section{Group model building: evaluations and observations}

To assess the impact of group model building, we analyzed observations and evaluations from the 2 first project workshops where UML was intensively used for building up joint conceptual frameworks.

Several graphical notations for the graphical visualisation of the framework were discussed at project workshops and bilateral meetings. UML (unified modelling language) was finally adopted as the modelling notation for the project framework on transition towards AM. UML is not a running model itself. It is rather a notation system that allows documenting or specifying knowledge about objects, relations and associations, workflows and processes, responsibilities, information flows, interfaces, etc. UML supports different views or frames on the same part of the world. All 'views' or diagrams share the same terminology and their level of detail depends on the degree of information that is required to understand a certain problem. Due to this approach a diagram is a view into a model presented from the aspect of a particular perspective (e.g. a stakeholder), it provides a partial representation of the system, and it is semantically consistent with other views.

Using UML in group discussions helps to make mutual assumptions explicit, because everybody attempts to translate his or her concepts into a common language. In selecting aspects, labelling them, drawing the relations and labelling the relations, differences between participants' frames can emerge and can be discussed. It also helps to keep the attention focussed on the developing diagram and it results in a tangible output of the discussion (one or more diagrams).

- "making these diagrams is useful to access knowledge that's in someone else's head"

- "with the diagrams you have to become more precise"

- "very useful for the discussion because it focuses the discussion on a visual image"

- "we were talking and talking until now but now we have a clear result"

- "it is positive that each of the groups made good products" 
Making the diagrams helps to identify where knowledge of a system or process is incomplete. Information about some elements or links may be missing.

- "I think it helps to identify knowledge gaps, to see where our description is incomplete"

- "It was interesting to see the bits of information that were lacking"

It's important to embed the making of diagrams in a larger process and clarify the goals of making them (knowledge representation, integration, facilitating discussions, ...). Otherwise, people may not see the point of making the diagrams, or at the other extreme, people may start using UML indiscriminately. Keeping track of the developed UML diagrams and limiting their number by prioritizing will not be an easy task.

- "The first big discussion was: how we can use these diagrams or why do we need them in the process, and if we now start with this are they really used later"

- "we should be clear about what we want to get out of it from the beginning so that we don't make a diagram for the sake of the diagram"

- "we should be very critical about which ones we make"

In terms of finding a mutually workable representation, the top level representation (the "matrix") itself may be the biggest challenge, since this captures world views on a high level of abstraction. In some cases, starting from representations of concrete subsystems may be easier (e.g. starting from the most important issues in a specific case).

- "We spent much time on the matrix and which cell was the most relevant for us. We ranked all the cells independently, and there were very different perceptions"

A workable equilibrium between technically correct UML and easily understandable UML should be found. The best diagrams may be those where "UML-experts" watch the formal correctness, while "UML-laypersons" assure it is easily understandable for non-experts as well.

- "formally correct UML diagrams are not necessarily the most easily understandable ones"

- $\quad$ "we need set quality standards, if not we are prone to ambiguities in interpretation, we need quality check"

A characteristic of UML as a formal language is that the visual lay-out of elements and relations in a specific view is technically meaningless: as long as the entities, attributes and relationships remain the same, you can rearrange the visual representation as you like without affecting what it means in UML. People are used however to derive meaning from the visual aspects of a diagram (above-below, leftright, close-distant, ...), and this may affect the meanings that are connected to the diagram. The advantage is that UML does not rely of these often implicit and possibly diverging meanings of the visual aspects of the diagram. The disadvantage is that people may still read those meanings into the visual aspects of the diagram and make diverging conclusions.

A general concern with respect to the integration of different theories into a conceptual model, is the difficult distinction between (1) connecting concepts that represent different parts of reality, and (2) connecting concepts that categorize differently the same part of reality. The problem is that our concepts to some extent define what we take to be the reality. The more cautious approach may be to allow for parallel representations of parts of reality where or when necessary (different ways of framing the issue), and try to identify overlapping parts and look for complementarities where possible. 


\section{Using concrete case contexts: evaluations and observations}

To analyze the impact of using concrete case contexts we used evaluations and observations from 3 project workshops. The third workshop was explicitly designed to profit as much as possible from the use of concrete case contexts.

The idea of using concrete case contexts when confronted with diverging theoretical frameworks, is that the meaning of a concept, theory or method can be easier understood when we look at specific contexts and illustrate there what the concept means. The concrete case context can then provide the necessary common ground to discuss concepts that come from different backgrounds.

In one of the facilitated workshops, case presentations were deliberately used for clarifying concepts related to AM. Two people who were each very familiar with a specific case, prepared an overview of the situation. The other participants were then invited to draw upon these inputs, and the presenters, to clarify and discuss the various concepts. A number of evaluations which were voiced during and at the end of the workshop referred to this approach, evaluating it as motivating and helpful.

- $\quad$ "we need examples for understanding what we mean, we may not have enough case studies in the project”

- $\quad$ "the early exemplary case approach was very helpful, it was good to have case study people around"

- $\quad$ "the case example taught me about complex adaptive systems, I understood better the meaning of transition as a natural process versus change as a result of deliberative actions"

- "the break-out groups on a very concrete case generated a lot of attention and energy, focusing on a concrete reality was enough to keep on contributing"

- "the case studies as background were useful, but could have been used even more"

However, concerns were also raised that scientific rigor may suffer when focusing on concrete cases.

- "NeWater is an Integrated Project and needs to develop integrated concepts first before applying it to cases. Otherwise the quality of generalizable scientific insights will suffer."

In another project workshop, efforts to integrate different approaches for vulnerability assessment didn't succeed. A direct comparison of different research methods seemed to be too sensitive. Probably the position of both research groups as well as the differing contexts in which these methods have been applied, made a comparison very difficult. The decision to choose one "stylized situation" on which the different approaches under investigation would be applied, created some commonality to easier interpret the differences in the results. This approach uses case contexts not in their full complexity and detail, but through a simplified representation. The stylized situation still refers to a specific situation in a specific case and is thus different from an abstracted theoretical model.

In the studied project, very different frames about uncertainty exist among scientists coming from different research traditions. In a workshop on uncertainty, concrete situations in which uncertainty was experienced by water management practitioners were collected through dialogue sessions with decisionmakers in several case study contexts. By means of a few open questions they were invited to tell about specific uncertainty related situations they had experienced, and these were summarized as short stories or vignettes. The most striking illustrations of different types of uncertainty were selected from the stakeholder dialogues and presented at workshop among scientists. Scientists from different fields discussed in break-out groups how they would deal with the uncertainties in specific case situations. In the discussion participants could easily refer back to these case situations, which provided a common focus for the group discussions. The cases allowed them to present and explain better their concepts and 
the approach they would take by applying them to the case examples. In the evaluations of this workshop, participants referred to this way of working in positive terms.

- "it was positive that it was linked to practical examples, so we could combine the different approaches"

- "it was quite good to work very concretely, in small groups"

- "it was very interesting to see that you get other views on the vignettes, from very different perspectives" 